\title{
Therapy of Liver Abscesses
}

\author{
Christoph Lübbert $^{\mathrm{a}}$ Johannes Wiegand $^{\mathrm{b}}$ Thomas Karlas $^{\mathrm{b}}$ \\ ${ }^{a}$ Division of Infectious Diseases and Tropical Medicine, Department of Gastroenterology and Rheumatology, Leipzig University Hospital, \\ Leipzig, Germany, \\ ${ }^{b}$ Department of Gastroenterology and Rheumatology, Leipzig University Hospital, Leipzig, Germany
}

\section{Keywords}

Liver abscess - Pyogenic liver abscess .

Klebsiella pneumoniae - Multidrug-resistant pathogens .

Amebic liver abscess - Entamoeba histolytica .

Antibiotics · Aspiration - Drainage

\section{Summary}

Background: Liver abscess (LA) is an uncommon but potentially life-threatening disease with significant morbidity and mortality. Methods: This review comprehensively describes epidemiology, pathogenesis, diagnosis, and treatment of LA, with a strong focus on antimicrobial treatment choices and the impact of multidrug-resistant pathogens. Results: In industrialized areas, pyogenic liver abscess (PLA) accounts for over $80 \%$ of the cases, whereas Entamoeba histolytica is responsible for up to $10 \%$ of the cases, with a higher incidence in tropical areas. Highly virulent strains of Klebsiella pneumoniae have emerged as a predominant cause of PLA in Asian countries and tend to spread to the USA, Australia, and European countries, therefore requiring special alertness. Most common symptoms of LA are fever, chills, and right upper quadrant abdominal pain, although a broad spectrum of non-specific symptoms may also occur. Conclusion: Imaging studies (ultrasound, computed tomography scan) and microbiological findings play a crucial role in the diagnosis of LA. The treatment of choice for PLA is a multimodal approach combining broad-spectrum antibiotics and aspiration or drainage of larger abscess cavities. Amebic LA can be cured by metronidazole therapy without drainage.

\section{Schlüsselwörter}

Leberabszess · Pyogener Leberabszess .

Klebsiella pneumoniae - Multiresistente Erreger . Amöbenleberabszess - Entamoeba histolytica . Antibiotika - Aspiration - Drainage

\section{Zusammenfassung}

Hintergrund: Leberabszesse (LA) sind eine seltene, jedoch potenziell lebensbedrohliche Erkrankung mit relevanter Morbidität und Mortalität. Methoden: Diese Übersicht beschreibt ausführlich Epidemiologie, Pathogenese, diagnostische Verfahren und Behandlung des LA. Der Schwerpunkt der Arbeit liegt dabei auf der Auswahl der geeigneten antibiotischen Therapie sowie des Managements bei Nachweis multiresistenter Erreger. Ergebnisse: Eitrige/pyogene Leberabszesse (PLA) sind mit über $80 \%$ die häufigste Entität in industrialisierten Gebieten, während Entamoeba histolytica in ca. 10\% der Fälle als Erreger identifiziert werden kann. In tropischen Gebieten ist die Inzidenz der Amöben-LA deutlich höher. Hochvirulente Stämme von Klebsiella pneumoniae haben sich in Asien zur Hauptursache der PLA entwickelt. Die zunehmende Verbreitung von K. pneumoniae auch in den USA, in Australien und in europäischen Ländern stellt eine Herausforderung für die Behandlung und Prävention von Sekundärkomplikationen dar. Typische Symptome bei LA sind Fieber, Schüttelfrost und rechtsseitiger Oberbauchschmerz, obgleich eine Vielzahl anderer, unspezifischer Symptome auftreten kann. Schlussfolgerung: Bildgebung (Ultraschall, Computertomographie) sowie mikrobiologische Untersuchungen sind geeignete diagnostische Verfahren bei klinischem Verdacht auf einen LA. Das multimodale Management des PLA beinhaltet die Therapie mit einem Breitspektrum-Antibiotikum sowie die Aspiration oder Drainage größerer Abszesse. Amöben-LA können durch Metronidazol geheilt werden und benötigen keine Drainage.

\section{KARGER \\ Fax +497614520714

\section{(C) 2014 S. Karger GmbH, Freiburg}

$1662-6664 / 14 / 0305-0334 \$ 39.50 / 0$
Dr. med. Christoph Lübbert

Division of Infectious Diseases and Tropical Medicine

Department of Gastroenterology and Rheumatology, Leipzig University Hospital

Liebigstraße 20, 04103 Leipzig, Germany

christoph.luebbert@medizin.uni-leipzig.de 


\section{Introduction}

Liver abscess (LA) is an uncommon but potentially lifethreatening disease with significant morbidity and mortality. LA have been described as far back as in ancient Greece in 400 B.C., when Hippocrates thought that prognosis of LA was related to the type of fluid in the lesion [1]. In 1890, Sir William Osler was the first to describe the presence of amebae in a patient's abscess and stools; however, it took until the early 20th century to conclude that Entamoeba histolytica was causally correlated to abscess formation in the liver [2]. In addition to pyogenic bacteria and E. histolytica, other pathogens, such as fungi and cytomegalovirus, can also cause LA, albeit rarely and especially in immunosuppressed patients.

In this review article, we comprehensively describe epidemiology, pathogenesis, diagnosis, and treatment of LA, with a strong focus on antimicrobial treatment choices and the impact of multidrug-resistant pathogens.

\section{Epidemiology}

In the first half of the last century and prior to the introduction of antibiotics, suppurative appendicitis was mainly responsible for the formation of pyogenic liver abscesses (PLA) $[2,3]$. In the last decades, however, hepatobiliary hitches have replaced portal pyemia and hematogenous spread as the most common cause of PLA [1-4]. The most relevant pathogens are Escherichia coli, Klebsiella spp., and Enterococcus spp. Among anaerobic bacteria, Bacteroides spp. and Fusobacterium spp. predominate $[1,2]$. Aerobic, anaerobic, or microaerophilic streptococci are isolated in $25-30 \%$ of cultures from PLA. In a high proportion of cases polymicrobial bacterial infection is recorded, with a strong etiological shift towards Klebsiella pneumoniae [1, 3, 4] (table 1, 2).

In the USA, the incidence of PLA is 8-15/100,000 inhabitants/year, accounting for over $80 \%$ of LA cases [1]. Abscesses due to E. histolytica account for up to $10 \%$ of the cases, and are more common in tropical areas as well as in tourists and in immigrants from developing countries, while fungi and other infectious agents are responsible for less than $10 \%$.

Before the 1980s, E. coli was the most common bacterial pathogen that caused PLA. During the past three decades, however, highly virulent strains of $K$. pneumoniae have emerged as a predominant cause in Asian countries, especially Taiwan [1-4]. The exact reason for this observation is not known, but it may be related to different host susceptibility to K. pneumoniae infection, expression of K. pneumoniaespecific virulence factors, higher $K$. pneumoniae carriage rates compared to the USA and Europe, and environmental factors [4]. For example, Chung et al. [5, 6] noted that people of Korean ethnicity who had lived in countries other than Korea had a lower proportion of carrying serotype K1 of K. pneumoniae strains than those who lived in Korea. These findings in-
Table 1. Microbiological findings in 63 patients with PLA who attended a tertiary hospital in Hong Kong between 1991 and 2001 (modified from [3])

\begin{tabular}{lll}
\hline & \multicolumn{2}{c}{ Number of cultures, \% } \\
\cline { 2 - 3 } & abscesses $(\mathrm{n}=44)$ & blood $(\mathrm{n}=46)$ \\
\hline Klebsiella & $26(43)$ & $22(35)$ \\
Escherichia coli & $12(20)$ & $13(20)$ \\
Streptococcus milleri & $7(12)$ & $5(8)$ \\
Anerobes & & \\
$\quad$ Bacteriodes fragilis & $6(10)$ & $8(13)$ \\
$\quad$ Peptostreptococcus & - & $2(3)$ \\
$\quad$ Clostridium sordelii & - & $1(1.5)$ \\
$\quad$ Fusobacterium & - & $3(1.5)$ \\
Enterococcus & $3(5)$ & $8(13)$ \\
Miscellaneous Gram- & $6(10)$ & $63(100)$ \\
$\quad$ negative organisms & & \\
Total isolates & $60(100)$ &
\end{tabular}

Table 2. Number of bacterial isolates recovered per case in patients with PLA who attended two municipal hospitals in New York City over a 10 -year period (modified from [1])

\begin{tabular}{ll}
\hline Number of isolates & Cases, \% \\
\hline 0 & 31.2 \\
1 & 44.2 \\
2 & 15.6 \\
3 & 6.5 \\
4 & 2.6 \\
\hline
\end{tabular}

dicate a potential role of environmental factors in intestinal colonization by these strains. To a lesser extent, the shift towards $K$. pneumoniae has also been observed in the USA, in Europe, and in Australia [1, 4, 7-10].

Middle-to-older-aged patients are at higher risk of developing PLA $[1,2]$. The peak incidence is found in 50- to 65-year-old patients, whereas PLA in children are rare. Male dominance is observed in patients with PLA, and the male to female ratio is approximately $1.5-2.5[1-4,11]$. According to the literature, mortality is ranging from $5-26 \%$ [1-5, 12-15]; however, owing to the improvement of diagnosis and treatment strategies, it has decreased in recent years and is now less than $10 \%[2,4]$.

Amebic LA was a relentlessly progressive and almost invariably fatal disease little more than a century ago, but since the introduction of effective medical treatment and rapid diagnosis, mortality rates have fallen to $1-3 \%$ [16].

\section{Etiology}

In contrast to other bacterial etiologies, PLA caused by $K$. pneumoniae are usually primary and cryptogenic. Since $K$. pneumoniae strains may colonize the human gastrointestinal tract, colonization is commonly anticipated to precede invasion of the intestinal mucosa and portal venous flow or as- 
cending biliary infection [4]. Some studies have suggested that translocation from the gastrointestinal tract is the most likely route by which $K$. pneumoniae causes formation of LA $[4,11]$. Fung et al. $[14,15]$ demonstrated that gastrointestinal carriage is a strong predisposing factor, and Lin et al. [17] reported a fecal carriage rate of K. pneumoniae in healthy adults of $75 \%$ and a high prevalence rate of serotype $\mathrm{K} 1 / \mathrm{K} 2$ isolates with the regulator of mucoid phenotype $\mathrm{A}$ ( $\mathrm{rmpA}$ ) plasmid gene among typical strains in Taiwan. In addition to intestinal colonization, cryptogenic invasive $K$. pneumoniae PLA are frequently associated with diabetes mellitus $[4,11,12]$, but do not show any clear association with peritoneal sources of infections, such as hepatobiliary obstruction, pancreatitis, enterocolitis, or malignomas $[2,4,11]$. Diabetes mellitus correlates with a high incidence of serotype $\mathrm{K} 1$ in $K$. pneumoniae PLA $[18,19]$. Poor glycemic control plays an important role in impairing the neutrophil phagocytic function of patients with K1/K2-type K. pneumoniae PLA [4, 11, 18-21].

Amebic LA arise from hematogenous spread (probably via the portal circulation) of amebic trophozoites that have breached the colonic mucosa [16]. Only few individuals presenting with amebic LA have concurrent amebic colitis, but the majority of patients have no bowel symptoms, and stool microscopy is usually negative for E. histolytica trophozoites and cysts.

\section{Clinical Manifestations}

Clinical presentations of patients with LA are not typical, and patients may present with vague constitutional symptoms. Most common are fever and chills, followed by abdominal pain restricted to the right upper quadrant, and hepatic tenderness [1-4]. Fever is a predominant symptom and has been reported in $90-95 \%$ of the cases [1-4] (table 3). A broad spectrum of non-specific symptoms like diarrhea, jaundice, right pleural effusion, anorexia, nausea, and vomiting may also occur $[1,11,15]$.

Although spontaneous rupture of LA has been rarely reported, there is a higher incidence of abscess rupture in the group of K. pneumoniae PLA patients compared to other LA etiologies $[11,12,20]$. Suggested risk factors for spontaneous rupture in $K$. pneumoniae PLA are persistent hyperglycemic dysregulation in diabetic patients, large abscess size $>5 \mathrm{~cm}$, and thinned-wall and gas-forming abscess $[4,7,11-13,15,19]$.

$K$. pneumoniae PLA is also associated with a higher likelihood of hematogenous spread and the potential for metastatic infection. The incidence rate of metastatic infection ranges from $10-45 \%$, especially in patients with diabetes mellitus due to impaired host-defense mechanisms [4, 5, 11, 12, 19-29]. Furthermore, an abscess size of $>5 \mathrm{~cm}$ has been described as a significant independent predictor [4, 11, 19-22]. Eyes, meninges, central nervous system (CNS), and lungs are the most common metastatic sites [7, 21-29]. Endophthalmitis is prob-
Table 3. Presenting features of 79 patients with PLA who attended two municipal hospitals in New York City over a 10-year period (modified from [1])

\begin{tabular}{ll}
\hline Symptoms & Cases, \% \\
\hline Fever & 89.6 \\
Right upper quadrant pain & 72.2 \\
Chills & 69.0 \\
Nausea & 43.1 \\
Vomiting & 32.3 \\
Weight loss & 26.1 \\
Jaundice & 21.4 \\
Headache & 17.5 \\
Myalgias & 11.9 \\
Diarrhea & 10.7 \\
\hline
\end{tabular}

ably the most serious septic complication of $K$. pneumoniae PLA, leading to subacute vision impairment. Affected patients usually do not recover their vision and may become entirely blind despite aggressive intravenous and intravitreal antibiotic treatment $[4,11,23,25,26,29]$. The mortality rate of $K$. pneumoniae PLA patients with evidence of metastatic infections is significantly higher than in patients without metastatic infections $[4,11,22,26]$.

Clinical presentation of patients with amebic LA is similar to those with PLA [16]. Individuals can present with amebic LA months or even years after travel or residency in an endemic area, so a careful travel history is mandatory. The disease should be suspected in anyone with an appropriate exposure history and fever, right upper quadrant pain, and substantial hepatic tenderness. Cough may be present, and dullness and rales in the right lung base are not infrequent. Jaundice is rather unusual. Symptoms are usually acute $(<10$ days in duration) but can be chronic, with anorexia and weight loss as prominent features [16].

\section{Laboratory Findings}

Anemia, leukocytosis, high erythrocyte sedimentation rate, elevated C-reactive protein level, hypoalbuminemia, and hyperbilirubinemia, as well as elevated alanine aminotransferase (ALAT) and alkaline phosphatase (AP) levels are the most common laboratory findings $[1,4,11]$. None of the blood tests specifically help to diagnose LA; however, they can suggest a liver abnormality that leads to targeted imaging studies.

In patients with amebic LA, leukocytosis without eosinophilia, mild anemia, raised concentrations of AP, and a high erythrocyte sedimentation rate are the most common laboratory observations [16].

\section{Imaging Findings}

LA are mainly located in the right lobe of the liver [1, 3, 4, $8,16,30]$. A high proportion of LA manifests itself as a solitary abscess, but multiple bilateral abscesses are also possible. 
Chest radiographs may reveal right-sided pulmonary infiltrates with pleural effusion $[1,3,4,11,30]$, and abdominal radiographs, which are rarely used but can be helpful in some cases, may show air-fluid levels or portal venous gas formation $[3,8,11,30]$. By now, ultrasound (US) and computed tomography (CT) are the two main diagnostic methods with a sensitivity of $96-100 \%[11,30]$.

On CT imaging, LA are of lower attenuation than the surrounding normal liver parenchyma (fig. 1). The abscess wall usually shows a rim enhancement on contrast-enhanced CT. The appearance of LA at US imaging is typically hypoechoic, but may also range from hyperechoic to hypoechoic (fig. 2) [30]. Gas-forming LA showing aerobilia have been rarely reported in PLA patients in the past; however, with the etiologic shift to $K$. pneumoniae as the primary causative agent, there is an increased risk of gas-producing PLA, especially in patients with diabetes mellitus failing glycemic control $[4,11]$.

Fig. 1. CT imaging of a large amebic LA in the right lobe of the liver.

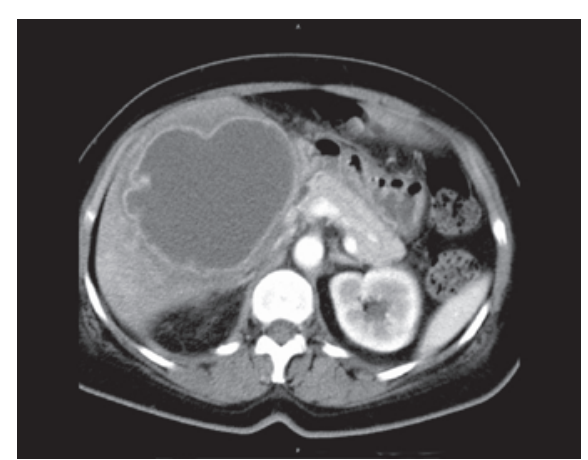

It is assumed that, under anaerobic conditions, K. pneumoniae strains being capable of facultative anaerobe metabolism can produce carbon dioxide by fermentation of glucose in tissue, especially under hyperglycemic conditions $[4,11]$.

\section{Diagnosis}

Diagnosis of LA is mainly based on imaging studies, paired with microbiological findings. CT scans have been reported to be more sensitive than US in the diagnosis of LA [11, 30]; however, from our own experience, both methods should be considered as equivalent. In terms of microbiological diagnosis, K. pneumoniae isolates obtained from blood or LA puncture with the hypermucoviscous phenotype is suggestive of an invasive $K$. pneumoniae strain, and the attending clinician should be notified as soon as possible by the microbiological laboratory [4]. Multiplex polymerase chain reaction (PCR) might be a useful rapid test for detection of the K. pneumoniae serotype that causes LA [31].

Metastatic infections are a clinical challenge and are only diagnosed in one third of the cases on admission [4]. Lungs, CNS, and eyes are the most common metastatic sites [4, 2129]. Thus, in diabetic patients, especially in Asians or those of Asian origin, who present with $K$. pneumoniae bacteremia, endophthalmitis, meningitis, or other extrahepatic infections, a search for an occult LA is indicated [4].

The diagnosis of amebic LA relies on liver imaging and positive amebic serology. Diagnostic fluid aspiration from the lesion is therefore not necessary (fig. 3, 4). US or CT scan are the
Fig. 2. Ultrasound morphology of LA (B mode): PLA frequently show an inhomogeneous hypoechoic pattern $(\mathbf{A})$ with a thickened, edematous wall (B, arrow). Blurred, irregular borders $(\mathbf{C})$ and septa (D) can also be observed. Morphology cannot discriminate between pyogenic $(\mathbf{A}, \mathbf{B}, \mathbf{D})$ and amebic (C) abscesses.
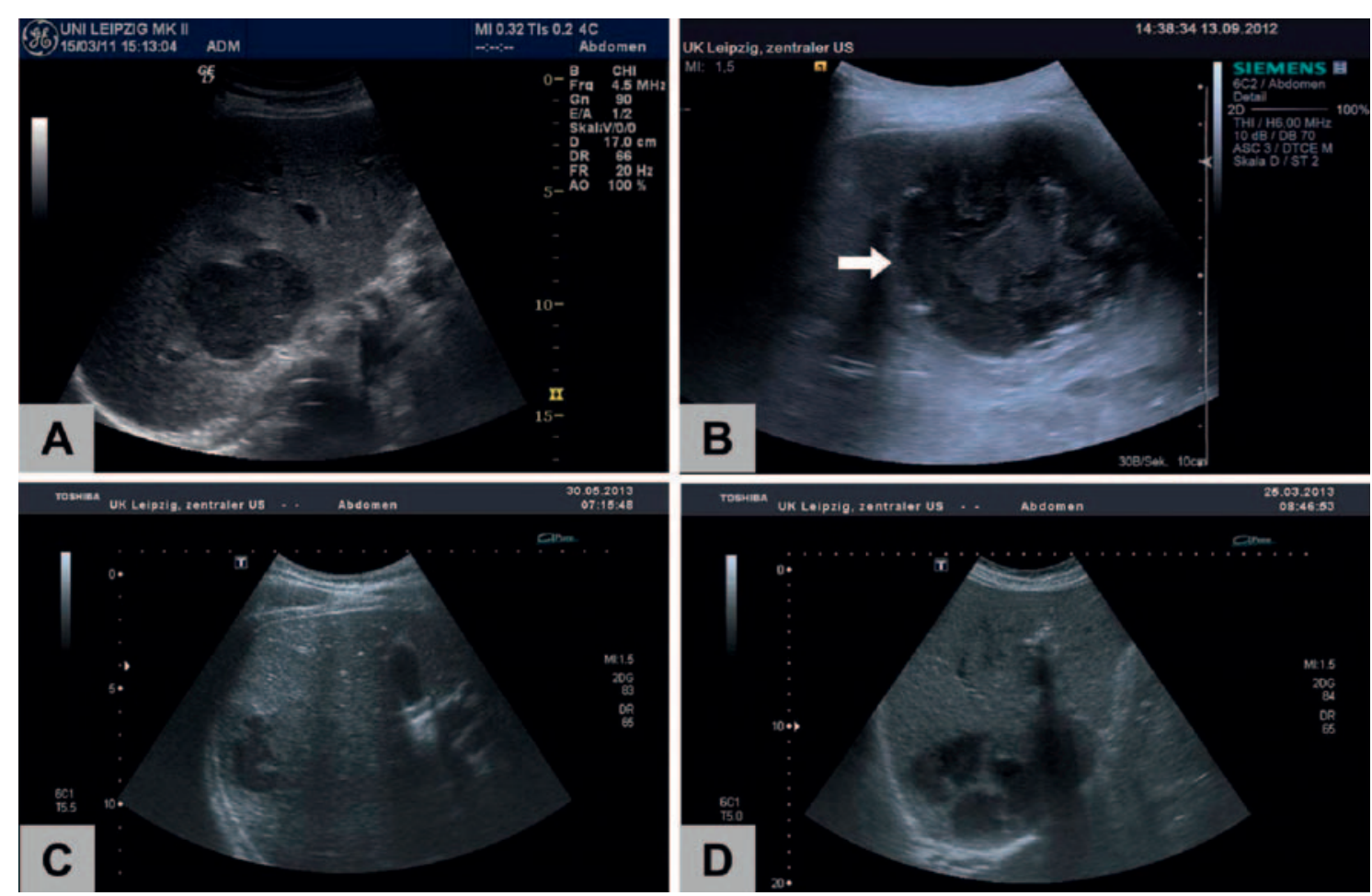
tests of choice; both methods are very sensitive, but neither provides absolute specificity for amebic LA [16]. Formerly, such abscesses were described as solitary lesions in the right lobe of the liver, but modern imaging techniques such as highresolution US or CT show a high frequency of multiple abscesses. Amebic serology is highly sensitive (>94\%) and highly specific $(>95 \%)$ for the diagnosis of amebic LA [16]. False-negative serological test results, however, can be obtained early in infection (within the first 7-10 days), but repeated tests will usually become positive. The successful use of an antigen detection enzyme-linked immunosorbent assay (ELISA) or PCR in patients with amebic LA suggests that these tests play an important part in the diagnosis of extraintestinal disease [16].

\section{Treatment}

\section{Antimicrobial Treatment of PLA}

First, it is essential to exclude amebic LA as a differential diagnosis. When the diagnosis of PLA is suspected, there is a need to start broad-spectrum antibiotics immediately after collection of microbiological specimen (obtained from abscess puncture and blood cultures) to control ongoing bacteremia and its associated complications $[4,11]$.

Recent studies have shown that most pathogens found in PLA are now resistant to ampicillin, but depending on the local resistance epidemiology, fluoroquinolones, third- and fourth-generation cephalosporins, piperacillin/tazobactam, aminoglycosides, and carbapenems remain effective treatment options [1, 4, 11, 32-34]. Preceding antibiotic therapy with ampicillin/amoxicillin has been described as a risk factor for K. pneumoniae PLA manifestation in Asia due to selection pressure [32]. Taking into account resistance rates against fluoroquinolones of up to $30 \%$ in E. coli, K. pneumoniae, and other enterobacteriaceae, third-generation cephalosporins (ceftriaxone and cefotaxime) and piperacillin/tazobactam have backed up a dominant position in the antibiotic treatment of PLA [1, 4, 8, 11, 32, 33]. Initial antibiotic regimens should therefore comprise either a third-generation cephalosporin and metronidazole or piperacillin/tazobactam, with the advantage of the latter variant that enterococcal infections are also partly covered.

When switching to de-escalation strategies, the selection of antimicrobials should be based on in vitro susceptibilities and clinical response. In most cases, targeted monotherapy with a beta-lactam tested active in vitro is sufficient. Combination therapy has been advocated for the treatment of bacteremic infections. There is current evidence that combination therapy with a beta-lactam and an aminoglycoside agent should be preferred in severely ill patients with Klebsiella spp. infections who experience hypotension [35]. Although PLA caused by ESBL(extended-spectrum beta-lactamase)-producing $K$. pneumoniae have been reported in Taiwan, such strains still form a minority [4, 11, 32-34]. Carbapenems are the drug of

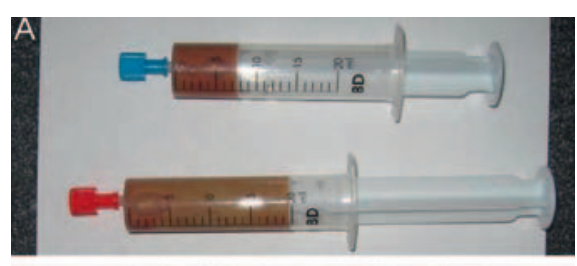

Fig. 3. Creamy brownish aspiration fluid of a PLA, together with small traces of blood $(\mathbf{A}, \mathbf{B})$.

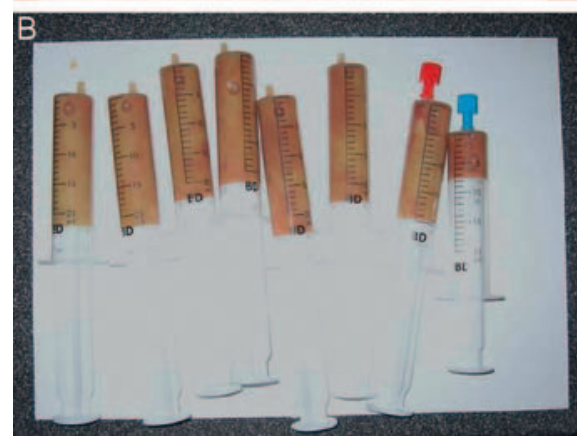

Fig. 4. Pink-colored aspiration fluid from a large amebic LA in a 46-year-old patient returning from an 8-week backpacking trip to India (courtesy Prof. Stefan Schubert, Leipzig University Hospital, Germany).

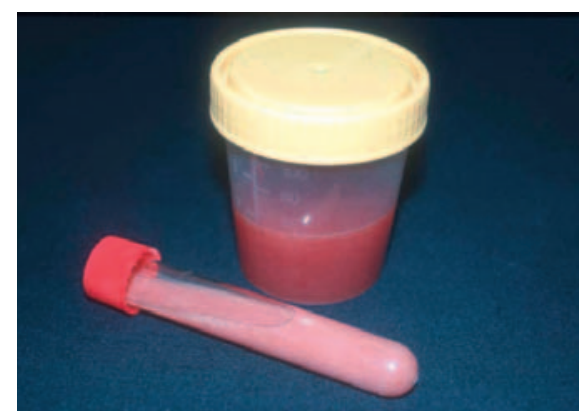

choice for ESBL-producing E. coli and K. pneumoniae. Carbapenem use (primarily imipenem/cilastatin) has been found to be independently associated with lower mortality than other antibiotics [4]. The growing incidence of carbapenemresistant K. pneumoniae, such as strains producing NDM-1 (New Delhi metallo-beta-lactamase-1) or KPC (K. pneumoniae carbapenemases), is of serious concern because of the few remaining treatment options (mainly tigecycline, colistin, and aminoglycosides) for these hyperresistant strains, which are associated with excess mortality [36-39].

The duration of therapy must be determined by response to treatment, as shown by repeated US of the abscess (fig. 5) and resolution of fever and leukocytosis [4]. The optimal duration of intravenous antimicrobial therapy, as well as the duration of subsequent oral therapy, remains unclear and does largely depend on the success rates of interventional source control. In studies from Taiwan, therapy generally consisted of 3 weeks of intravenous antibiotics followed by 1-2 months of oral therapy $[4,11]$. However, our own experience as well as studies performed in the USA indicate that shorter courses of antibiotics with targeted intravenous therapy for 2-3 weeks and consecutive oral therapy for 1-2 weeks are associated with extremely low mortality of less than $5 \%[1,8]$.

Metastatic infections of the CNS and eyes in K. pneumoniae PLA are severe and difficult to treat. In the absence of 
Fig. 5. Repeated ultrasound imaging shows successful treatment of a PLA in the right lobe of the liver in a 54-year-old male patient: Under combined treatment with needle aspiration and antibiotics a 'meltdown' (A-D) of the abscess (initial size: $4.0 \times 2.9 \mathrm{~cm}$ ) could be demonstrated within 4 weeks.


ESBL-producing strains, third-generation cephalosporins are the drugs of choice for $K$. pneumoniae meningitis in view of their better penetration into the cerebrospinal fluid (compared with first-generation and second-generation cephalosporins) [4]. Both cefotaxime and ceftriaxone are effective for the treatment of meningitis. Large doses are used for both intravenous cefotaxime (up to $2 \mathrm{~g}$ every $4 \mathrm{~h}$ ) and ceftriaxone ( $2 \mathrm{~g}$ twice a day). 3 weeks of treatment has been recommended because of a high rate of relapse in individuals treated with shorter treatment courses [4]. Imipenem/cilastatin or meropenem should be given to patients instead of third-generation cephalosporins when ESBL-producing strains are suspected.

Both intravitreal and intravenous routes should be used for treatment of $K$. pneumoniae endophthalmitis. Intravenous ceftazidime plus amikacin has been the most widely used combination [4]. Combination intravitreal treatment with cephalosporins (cefazolin and ceftazidime) and aminoglycosides (gentamicin and amikacin) have been applied successfully [4].

Furthermore, glycemic control in diabetic patients plays an essential role in the clinical features of $K$. pneumoniae PLA, especially in metastatic complications [4].

\section{Interventional Therapy of PLA}

Percutaneous drainage was widely used during the past three decades due to the obvious advantages of simplicity and avoidance of general anesthesia and laparotomy [1, 4, 8, 11]. Approaching multiple PLA, percutaneous drainage may be associated with a higher failure rate. However, a recent retrospective study showed that treatment with percutaneous transhepatic drainage demonstrated similar effectiveness for patients with multiple abscesses but shorter hospitalization when compared with surgical drainage, which suggested that percutaneous aspiration or drainage should always be undertaken before surgery in terms of its lower morbidity and less cost [40].

Yu et al. [41] found that intermittent needle aspiration was probably as effective as continuous catheter drainage for the treatment of PLA. Due to the solid nature of $K$. pneumoniae PLA, procedure simplicity, patient comfort, and reduced costs, needle aspiration deserves to be considered as a firstline drainage approach in this disease entity, if not in all PLA. Patients with the following criteria have been suggested for percutaneous drainage: patients who continued to be febrile even after 48-72 h of adequate medical and aspiration treatment; LA > $6 \mathrm{~cm}$ in size; and clinical or US features suggesting impending perforation [11].

Common material for US-guided aspiration or drainage of LA is shown in figure 6.

\section{Surgical Interventions in Patients with PLA}

Surgical drainage has been reserved for patients who fail to respond to treatment with either aspiration or percutaneous drainage and antibiotics or who have concurrent intra-abdominal pathology which requires surgical management $[1,4,41$, 42]. In addition to conventional laparotomy, laparoscopic drainage in association with systemic antibiotic therapy has been described as a safe and effective minimally invasive approach [2]. Thus, percutaneous and surgical techniques are not competing methods, but they have different indications, and surgery also represents an option for non-responders to percutaneous treatment $[2,11,42]$. 


\section{Management of Amebic LA}

Due to the excellent response to metronidazole, the management of amebic LA is unique. Abscesses occupying large areas of the liver can be cured without drainage, even by one dose of metronidazole [16]. Most patients show a response to treatment (reduced fever and abdominal pain) within 72-96 h. Individuals with amebic LA should also be treated with a luminal agent (diloxanide furoate or paromomycin) to eliminate intestinal colonization by E. histolytica.

Surgical drainage of uncomplicated amebic LA is generally unnecessary and should be avoided [16]. The role of percutaneous therapeutic aspiration guided by US or CT in the treatment of uncomplicated amebic LA remains controversial [16]. Clearly, most such abscesses can be cured by metronidazole treatment alone, and in a randomized controlled study no significant difference in either length of hospitalization or duration of time to becoming afebrile was seen between individuals treated with metronidazole alone and those treated with metronidazole as well as percutaneous therapeutic aspiration $[16,43]$. However, a prospective study in which large amebic LA underwent aspiration, while small abscesses were treated with drugs alone, showed benefits in mean hospital stay and the speed of clinical improvement in the aspiration group [16].

A reasonable policy might be to reserve aspiration for individuals in whom diagnosis is uncertain (where PLA or bacterial superinfection of the amebic LA is a concern), those who do not respond to metronidazole (persistent fever or abdominal pain after 4 days of treatment), individuals with large left lobe abscesses (because of the risk of rupture into the pericardium), and severely ill patients in whom an accelerated clinical course and large abscesses make rupture seem imminent [16]. Aspiration, percutaneous catheter drainage, or both improve outcomes in the treatment of amebic empyema after abscess rupture, and percutaneous catheter (or, if necessary, surgical) drainage could be lifesaving in the treatment of amebic pericarditis [16]. Amebic LA rupture into the peritoneum might also respond best to conservative treatment, medical management, and use of percutaneous catheter drainage if localized collections of fluid are noted [16, 43].

\section{Conclusion}

LA remain an important and potentially life-threatening disease. However, the mortality rate in recent decades has been significantly reduced and is today less than $10 \%$ in PLA, in amebic LA even below 3\%.

During the last three decades, highly virulent strains of $K$. pneumoniae (serotypes $\mathrm{K} 1$ and $\mathrm{K} 2$ with the rmpA plasmid gene) have emerged as a predominant cause of PLA in Asian countries, especially Taiwan, and this trend has been observed to a lesser extent also in the USA, in Europe, and in Australia. Therefore, corresponding changes in pathogen etiologies must be globally taken into account when setting diagno-

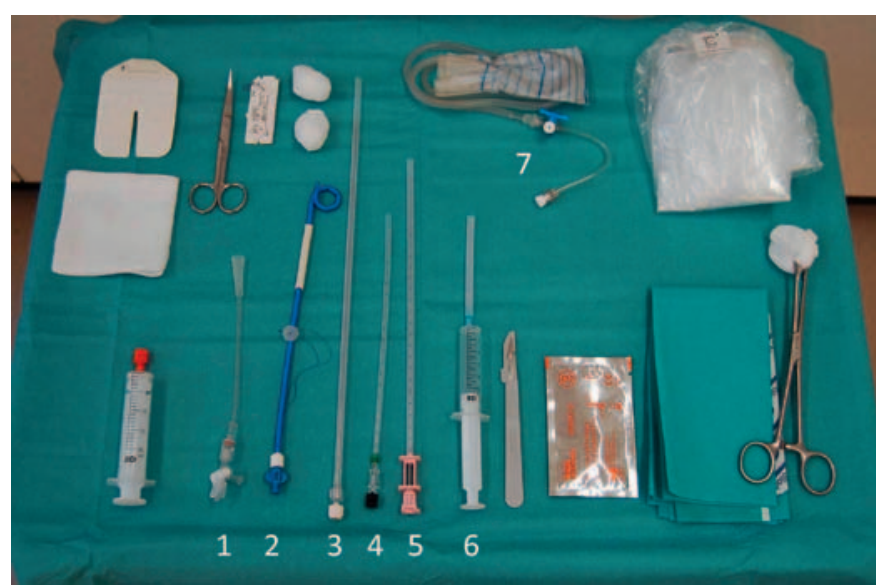

Fig. 6. Material for interventional therapy of LA: For drainage, a pigtail catheter (2) is placed into the abscess cavity using a guide needle (3). An adaptor (1) connects the catheter with a drainage bag (7). Alternatively, different fine needle types (4-6) can be used for aspiration of liquid abscess content.

sis and treatment of PLA. Diabetes mellitus and serotype K1 infection are the most common risk factors for recurrent $K$. pneumoniae PLA. The prognosis for patients with endophthalmitis caused by $K$. pneumoniae is still very poor, with more than $85 \%$ of the patients retaining a severe visual deficit.

The therapeutic success in PLA can be ensured best in a multimodal approach with inclusion of antibiotics, US- or CTassisted aspiration or drainage, and surgical relief as a last option. According to our own experience, needle aspiration should be preferred, taking into account increased patient discomfort and substantial dislocation rates especially when dorsal or dorsolateral drainage is applied.

In consideration of current resistance rates reaching up to $90 \%$ against ampicillin and up to $30 \%$ against fluoroquinolones in most enterobacteriaceae, third-generation cephalosporins (combined with metronidazole) and piperacillin/tazobactam have obtained a dominant position in the antibiotic treatment of PLA. Fortunately, PLA caused by ESBL-producing $K$. pneumoniae still form a minority; however, ESBL-producing strains are globally on the rise. Carbapenems are the drug of choice for ESBL-producing E. coli and K. pneumonia, and their use (primarily imipenem/cilastatin) has been found to be independently associated with lower mortality than other antibiotics.

Due to the excellent response to antiparasitic therapy with metronidazole, the management of amebic LA is different from PLA, with the result that even amebic LA occupying large areas of the liver can be cured without drainage.

\section{Disclosure Statement}

There was no funding. All authors indicate no potential conflicts of interest. 


\section{References}

- 1 Rahimian J, Wilson T, Oram V, Holzman RS: Pyogenic liver abscess: recent trends in etiology and mortality. Clin Infect Dis 2004;39:1654-1659.

2 Romano G, Agrusa A, Frazzetta G, et al: Laporoscopic drainage of liver abscess: case report and review of the literature. G Chir 2013;34:180-182.

3 Wong WM, Wong BC, Hui CK, et al: Pyogenic liver abscess: retrospective analysis of 80 cases over a 10-year study period. J Gastroenterol Hepatol 2002;17:1001-1007.

4 Siu LK, Yeh KM, Lin JC, et al: Klebsiella pneumoniae liver abscess: a new invasive syndrome. Lancet Infect Dis 2012;12:881-887.

5 Chung DR, Lee SS, Lee HR, et al.; and the Korean Study Group for Liver Abscess: Emerging invasive liver abscess caused by K1 serotype Klebsiella pneumoniae in Korea. J Infect 2007;54:578-583.

6 Chung DR, Lee H, Park MH, et al: Fecal carriage of serotype K1 Klebsiella pneumoniae ST23 strains closely related to liver abscess isolates in Koreans living in Korea. Eur J Clin Microbiol Infect Dis 2012;31:481-486.

7 Lederman ER, Crum NF: Pyogenic liver abscess with a focus on Klebsiella pneumoniae as a primary pathogen: an emerging disease with unique clinical characteristics. Am J Gastroenterol 2005;100:322331.

8 Pastagia M, Arumugam V: Klebsiella pneumoniae liver abscesses in a public hospital in Queens, New York. Travel Med Infect Dis 2008;6:228-233.

$\checkmark 9$ Moore R, O'Shea D, Geoghegan T, Mallon PW, Sheehan G: Community-acquired Klebsiella pneumoniae liver abscess: an emerging infection in Ireland and Europe. Infection 2013;41:681-686.

10 Chavada R, Ng J, Maley M, Descallar J: Emergence of Klebsiella pneumoniae liver abscesses in South-western Sydney. Infection 2014;42:595-596.

11 Liu Y, Wang JY, Jiang W: An increasing prominent disease of Klebsiella pneumoniae liver abscess: etiology, diagnosis, and treatment. Gastroenterol Res Pract 2013;2013:258514.

12 Yang CC, Yen CH, Ho MW, Wang JH: Comparison of pyogenic liver abscess caused by non-Klebsiella pneumoniae and Klebsiella pneumoniae. J Microbiol Immunol Infect 2004;37:176-184.

13 Chen SC, Wu WY, Yeh CH, et al: Comparison of Escherichia coli and Klebsiella pneumoniae liver abscesses. Am J Med Sci 2007;334:97-105.

14 Fung CP, Siu LK: Virulence of Klebsiella pneumoniae serotype $\mathrm{K} 2$ should not be underestimated in $K$ pneumoniae liver abscess. Clin Infect Dis 2007; 45:1530-1531, author reply 1532-1533.

15 Fung CP, Lin YT, Lin JC, et al: Klebsiella pneumoniae in gastrointestinal tract and pyogenic liver abscess. Emerg Infect Dis 2012;18:1322-1325.

16 Stanley SL Jr: Amebiasis. Lancet 2003;361:10251034.
17 Lin YT, Siu LK, Lin JC, et al: Seroepidemiology of Klebsiella pneumoniae colonizing the intestinal tract of healthy Chinese and overseas Chinese adults in Asian countries. BMC Microbiol 2012;12:13.

$18 \mathrm{Yu}$ WL, Chan KS, Ko WC, Lee CC, Chuang YC: Lower prevalence of diabetes mellitus in patients with Klebsiella pneumoniae primary liver abscess caused by isolates of $\mathrm{K} 1 / \mathrm{K} 2$ than with non-K1/K2 capsular serotypes. Clin Infect Dis 2007;45:15291530, author reply $1532-1533$.

19 Kim JK, Chung DR, Wie SH, et al: Risk factor analysis of invasive liver abscess caused by the K1 serotype Klebsiella pneumonia. Eur J Clin Microbiol Infect Dis 2009;28:109-111.

$20 \mathrm{Yu}$ WL, Ko WC, Cheng KC, Lee CC, Lai CC, Chuang YC: Comparison of prevalence of virulence factors for Klebsiella pneumoniae liver abscesses between isolates with capsular $\mathrm{K} 1 / \mathrm{K} 2$ and non-K1/K2 serotypes. Diagn Microbiol Infect Dis 2008;62:1-6.

$21 \mathrm{Yu}$ WL, Hansen DS, Ko WC, et al.; and the International Klebsiella Study Group: Virulence characteristics of Klebsiella and clinical manifestations of $K$ pneumoniae bloodstream infections. Emerg Infect Dis 2007;13:986-993.

22 Lee SS, Chen YS, Tsai HC, et al: Predictors of septic metastatic infection and mortality among patients with Klebsiella pneumoniae liver abscess. Clin Infect Dis 2008;47:642-650.

23 Liu YC, Cheng DL, Lin CL: Klebsiella pneumoniae liver abscess associated with septic endophthalmitis. Arch Intern Med 1986;146:1913-1916.

$24 \mathrm{Hu}$ BS, Lau YJ, Shi ZY, Lin YH: Necrotizing fasciitis associated with Klebsiella pneumoniae liver abscess. Clin Infect Dis 1999;29:1360-1361.

25 Casanova C, Lorente JA, Carrillo F, Perez-Rodriguez E, Nunez N: Klebsiella pneumoniae liver abscess associated with septic endophthalmitis. Arch Intern Med 1989;149:1467.

26 Fang CT, Lai SY, Yi WC, Hsueh PR, Liu KL, Chang SC: Klebsiella pneumoniae genotype K1:an emerging pathogen that causes septic ocular or central nervous system complications from pyogenic liver abscess. Clin Infect Dis 2007;45:284-293.

27 Tang LM, Chen ST, Hsu WC, Chen CM: Klebsiella meningitis in Taiwan: an overview. Epidemiol Infect 1997;119:135-142.

28 Yang PW, Lin HD, Wang LM: Pyogenic liver abscess associated with septic pulmonary embolism. J Chin Med Assoc 2008;71:442-447.

29 Yang CS, Tsai HY, Sung CS, Lin KH, Lee FL, Hsu WM: Endogenous Klebsiella endophthalmitis associated with pyogenic liver abscess. Ophthalmology 2007;114:876-880.

30 Lin AC, Yeh DY, Hsu YH, et al: Diagnosis of pyogenic liver abscess by abdominal ultrasonography in the emergency department. Emerg Med J 2009; 26:273-275.
1 Turton JF, Baklan H, Siu LK, Kaufmann ME, Pitt TL: Evaluation of a multiplex PCR for detection of serotypes K1, K2 and K5 in Klebsiella spp. and comparison of isolates within these serotypes. FEMS Microbiol Lett 2008;284:247-252.

32 Lin YT, Liu CJ, Yeh YC, et al: Ampicillin and amoxicillin use and the risk of Klebsiella pneumonia liver abscess in Taiwan. J Infect Dis 2013;208: 211-217.

33 Lin JC, Siu LK, Fung CP, Yeh KM, Chang FY: Nosocomial liver abscess caused by extended-spectrum beta-lactamase-producing Klebsiella pneumoniae. J Clin Microbiol 2007;45:266-269.

34 Su SC, Siu LK, Ma L, et al: Community-acquired liver abscess caused by serotype K1 Klebsiella pneumoniae with CTX-M-15-type extended-spectrum beta-lactamase. Antimicrob Agents Chemother 2008;52:804-805.

35 Korvick JA, Bryan CS, Farber B, et al: Prospective observational study of Klebsiella bacteremia in 230 patients: outcome for antibiotic combinations versus monotherapy. Antimicrob Agents Chemother 1992;36:2639-2644.

36 Nordmann P, Cuzon G, Naas T: The real threat of Klebsiella pneumoniae carbapenemase-producing bacteria. Lancet Infect Dis 2009;9:228-236.

37 Munoz-Price LS, Poirel L, Bonomo RA, et al: Clinical epidemiology of the global expansion of Klebsiella pneumoniae carbapenemases. Lancet Infect Dis 2013;13:785-796.

38 Lübbert C, Faucheux S, Becker-Rux D, et al: Rapid emergence of secondary resistance to gentamicin and colistin following selective digestive decontamination in patients with KPC-2-producing Klebsiella pneumoniae: a single-centre experience. Int J Antimicrob Agents 2013;42:565-570.

39 Kumarasamy KK, Toleman MA, Walsh TR, et al: Emergence of a new antibiotic resistance mechanism in India, Pakistan, and the UK: a molecular, biological, and epidemiological study. Lancet Infect Dis 2010;10:597-602.

40 Ferraioli G, Garlaschelli A, Zanaboni D, et al: Percutaneous and surgical treatment of pyogenic liver abscesses: observation over a 21-year period in 148 patients. Dig Liver Dis 2008;40:690-696.

41 Yu SC, Ho SS, Lau WY, et al: Treatment of pyogenic liver abscess: prospective randomized comparison of catheter drainage and needle aspiration. Hepatology 2004;39:932-938.

42 Hsieh HF, Chen TW, Yu CY, et al: Aggressive hepatic resection for patients with pyogenic liver abscess and APACHE II score $\geq 15$. Am J Surg 2008; 196:346-350.

43 Allan RJ Van, Katz MD, Johnson MB, et al: Uncomplicated amebic liver abscess: prospective evaluation of percutaneous aspiration. Radiology 1991; 183:827-830. 